\title{
Modelagem da hidrógrafa de cheia em uma bacia hidrográfica da região Alto Rio Grande
}

\author{
Polyanna M. de O. Silva ${ }^{1}$, Carlos R. de Mello ${ }^{1}$, Antônio M. da Silva ${ }^{1}$ \& Gilberto Coelho ${ }^{1}$
}

\begin{abstract}
RESUMO
O escoamento superficial não é apenas o principal componente do ciclo hidrológico associado à erosão hídrica, mas também é responsável pelo transporte dos sedimentos. Dada a sua importância, diversos modelos hidrológicos foram desenvolvidos para melhor se entender este processo e representar o comportamento das bacias hidrográficas; objetivou-se, assim, avaliar a aplicação do Modelo Conceitual de Nash para geração do Hidrograma Unitário Instantâneo (HUI), aos dados de escoamento superficial na sub-bacia hidrográfica do Ribeirão Marcela. O HUI, gerado pelo Modelo Conceitual de Nash, apresentou boa precisão para modelagem do escoamento superficial nesta sub-bacia hidrográfica.
\end{abstract}

Palavras-chave: modelo de Nash, deflúvio, hidrograma unitário

\section{Modeling of the flood hydrograph in a watershed of the Alto Rio Grande region}

\begin{abstract}
Surface runoff is one of the most important hydrological cycle components associated with water erosion, being responsible for sediment transport. Several hydrological models have been developed for a better understanding of this process and for representing the hydrological behavior of the watershed. The objective of this study is to analyze the Nash Conceptual Model for Instantaneous Unit Hydrograph (IUH) generating the flow behavior in the Ribeirão Marcela Watershed, Alto Rio Grande region. The IUH generated by the Nash Conceptual Model presents a good accuracy for the modeling of surface runoff hydrograph in this watershed.
\end{abstract}

Key words: Nash's modeling, surface runoff, unit hydrograph 


\section{INTRODUÇÃO}

O escoamento superficial é o seguimento do ciclo hidrológico que estuda o deslocamento das águas na superfície da Terra, considerado um dos mais importantes para o manejo da bacia hidrográfica; pode ser dividido em componentes, com destaque para o escoamento superficial direto e o escoamento de base ou subterrâneo. O primeiro componente resulta da parcela da precipitação que excede a capacidade de infiltração do solo e escoa sobre a sua superfície, gerando cheias; é freqüente quando ocorrem precipitações muito intensas e/ou quando o solo já está muito umedecido e com capacidade de infiltração reduzida. Esta parcela do escoamento é conhecida como precipitação efetiva ou deflúvio superficial e sua importância está diretamente associada a dimensionamentos hidráulicos, como barragens, terraços, bacias de contenção e controle da erosão hídrica (Tucci, 2001; Pruski et al., 2003). O escoamento de base ou subterrâneo é aquele produzido pela drenagem do aqüífero subterrâneo, importante do ponto de vista ambiental, uma vez que refletirá a produção de água na bacia durante as estações secas (Tucci, 2001).

A hidrologia no contexto de bacias hidrográficas busca conceituar e modelar diferentes processos que interagem sob condições ambientais variáveis no espaço e no tempo (Schuler, 2003); assim, a simulação hidrológica é de substancial significância para a previsão de respostas hidrológicas dessas unidades ambientais a determinado evento de precipitação (Tucci \& Collischonn, 2003; Collischonn, 2001).

De acordo com Quintela \& Portela (2002) os modelos podem ser classificados como determinísticos, baseados na explicação física do fenômeno, podendo ser empírico, conceitual ou semi-conceitual e não determinísticos, com base em modelos probabilísticos ou estocásticos, cujos fenômenos não são explicados por meio de outras variáveis físicas. Dentre os modelos conceituais de base física que representam o escoamento superficial, pode-se destacar o modelo desenvolvido por Nash (1957), que representa o escoamento na bacia por $n$ reservatórios em cascata e considera comportamento linear entre este e o armazenamento nos reservatórios (Agirre et al., 2005; Tucci, 1998).

Objetivou-se, com o estudo, mediante esses aspectos, avaliar a aplicação do Modelo Conceitual de Nash para modelamento do hidrograma de escoamento superficial na bacia hidrográfica do ribeirão Marcela, Alto Rio Grande, MG.

\section{MATERIAL E MÉTODOS}

\section{Monitoramento hidroclimático e caracterização da bacia hidrográfica}

A área de estudo corresponde à bacia hidrográfica do Ribeirão Marcela, afluente pela margem direita do córrego Jaguara, que deságua diretamente no reservatório da Usina Hidrelétrica de Camargos/CEMIG; situa-se entre as coordenadas UTM 550169 e 552810 W e 7650163 e 7650989 S, fuso $23 \mathrm{k}$, datum Córrego Alegre, e entre as altitudes 960 e $1060 \mathrm{~m}$ e se acha inserida na unidade geomorfológica Pla- nalto Campo das Vertentes, na região Alto Rio Grande, município de São João Del Rei. Esta bacia hidrográfica é representativa do domínio dos Latossolos na região e foi escolhida com base em estudos pedológicos e hidrogeológicos, para monitoramento ambiental visando à simulação hidrológica (Silva \& Mello, 2005).

A sub-bacia hidrográfica possui área de drenagem de 478 ha, declividade média de 13,65\% e elevação média de 1003 m. O comprimento do curso d’água principal é de $3,83 \mathrm{~km}$ sendo a declividade do canal de $1,57 \%$ e a densidade de drenagem igual a $1,80 \mathrm{~km} \mathrm{~km}^{-2}$.

Os dados climatológicos foram monitorados por uma estação meteorológica completa, instalada na seção de controle da bacia hidrográfica, programada para fornecer dados a cada 15 ou 30 min, de acordo com a estação do ano. De posse das lâminas precipitadas no intervalo de tempo, construíramse os respectivos hietogramas para cada evento registrado.

O escoamento superficial foi monitorado por meio de uma estação fluviométrica contendo um linígrafo automático com sensor de pressão instalado em poço tranqüilizador, programado para fornecer o nível d'água conforme intervalos de tempo especificados. Os linigramas foram convertidos em hidrogramas, através da curva-chave gerada ao longo do tempo para a seção monitorada:

$$
\mathrm{Q}=0,5002 \cdot \mathrm{H}^{1,866}, \quad \mathrm{r}^{2}=0,978
$$

Sendo: $\mathrm{Q}$ a vazão em $\mathrm{m}^{3} \mathrm{~s}^{-1}$ e $\mathrm{H}$ o nível de água em $\mathrm{m}$.

Depois de construídos os hidrogramas promoveu-se a separação do escoamento superficial direto e do subterrâneo, determinando-se o deflúvio total, em mm, conforme Tucci (2001), considerando comportamento linear do escoamento subterrâneo durante a existência do escoamento superficial.

\section{Modelagem conceitual do hidrograma de cheia}

Nash (1957) representou o escoamento superficial direto em uma bacia hidrográfica por $n$ reservatórios lineares em cascata, cuja situação considera uma precipitação uniforme ao longo da bacia e sua propagação até a respectiva seção de controle. A consideração de reservatórios simula uma situação de amortização da vazão de pico e outra de translação, ou seja, de um movimento de ondas do escoamento cujo efeito é mais pronunciado em canais ao longo da bacia. Fisicamente, a facilidade com que o reservatório drena o escoamento é representada por um parâmetro k, o qual reflete a resposta da bacia hidrográfica ao ser excitada por um ou mais eventos de precipitação efetiva (Tucci, 1998; Fendrich, 1984). Tomando-se 2 reservatórios diferentes em série, com constantes k1 e k2, a saída do primeiro é a entrada do segundo, com a integral defasada de um intervalo de tempo $(\tau)$, proporcionando o Hidrograma Unitário Instantâneo (HUI), cuja equação geral é:

$$
\mathrm{u}(0, \mathrm{t})=\int_{\tau=0}^{\tau=\mathrm{t}} \frac{1}{\mathrm{k}_{1}} \mathrm{e}^{-\tau / \mathrm{k} 1} \cdot \frac{1}{\mathrm{k}_{2}} \mathrm{e}^{-(\mathrm{t}-\tau / k 2)} \mathrm{d} \tau
$$

Resolvendo-se a integral da Eq. 2 e se considerando 2 reservatórios iguais, obter-se-á: 


$$
\begin{aligned}
& u(0, t)=\int_{0}^{t} \frac{1}{k^{2}} \cdot \exp \left(\frac{-t}{k}\right) d \tau \\
& u(0, t)=\frac{t}{k^{2}} \cdot\left(e^{-t k}\right)
\end{aligned}
$$

Para 3 reservatórios:

$$
\mathrm{u}(0, \mathrm{t})=\frac{\mathrm{t}^{2}}{2 \times \mathrm{k}^{3}} \cdot\left(\mathrm{e}^{-\mathrm{tk}}\right)
$$

Generalizando:

$$
\mathrm{u}(0, \mathrm{t})=\frac{\mathrm{t}^{\mathrm{n}-1}}{\mathrm{k}^{\mathrm{n}} \times(\mathrm{n}-1) !} \cdot \mathrm{e}^{-\mathrm{tk}}
$$

A Eq. 6 pode ser representada, genericamente, da seguinte forma:

$$
\mathrm{u}(0, \mathrm{t})=\left(\frac{\mathrm{t}}{\mathrm{k}}\right)^{\mathrm{n}-1} \cdot \frac{\mathrm{e}^{-\mathrm{tk}}}{\mathrm{k} \cdot \Gamma(\mathrm{n})}
$$

A Eq. 7 é conhecida como modelo conceitual de Nash para HUI, considerando-se $n$ reservatórios lineares representando a situação esquematizada na Figura 1.

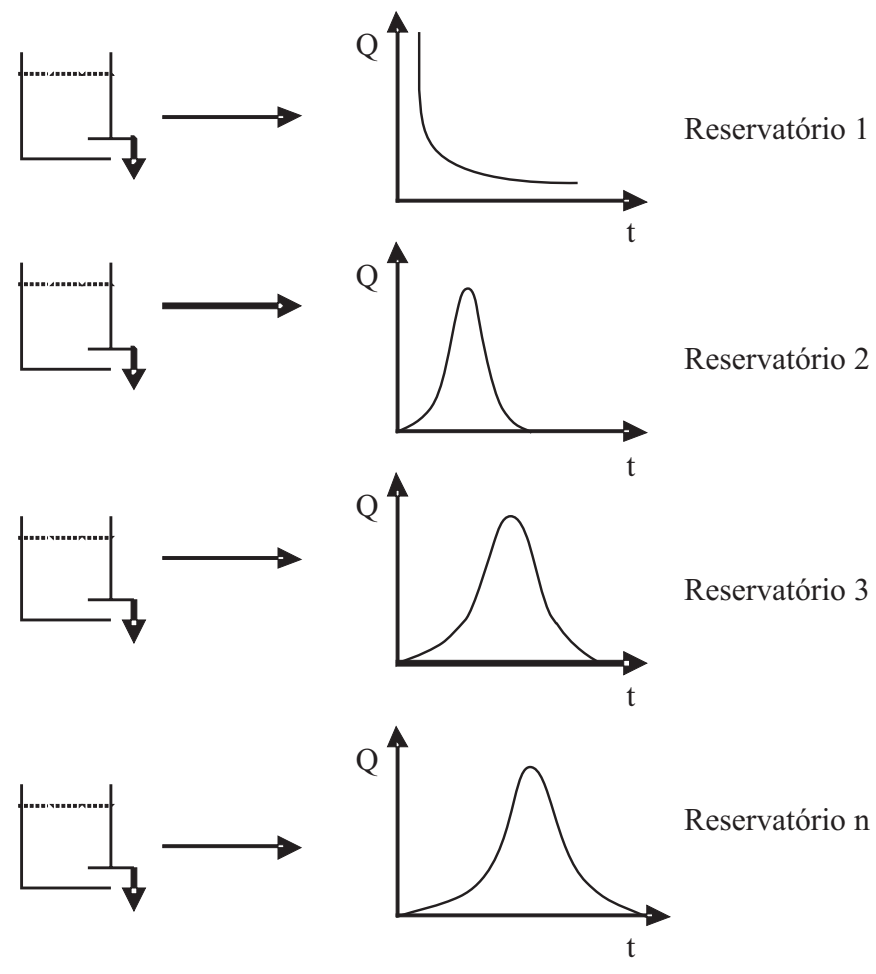

Figura 1. Representação esquemática do modelo de Nash para $n$ reservatórios lineares

A determinação da vazão Q(t), como função dos parâmetros n e k, é realizada por meio da equação de convolução do HUI, considerando-se primeiro a precipitação unitária, gerando as ordenadas do Hidrograma Unitário (HU) pela integração do HUI:

$$
h(t)=\int_{0}^{1} \frac{1}{k}\left(\frac{t-\tau}{k}\right)^{n-1} \cdot \frac{e^{-(t-\tau) / k}}{\Gamma(n)} d \tau
$$

Esta integral pode ser resolvida numericamente utilizando-se a regra de Simpson. Subdividindo-se o intervalo $(0,1)$ em $m$ intervalos, cada ordenada é obtida por:

$$
\mathrm{ht}=\frac{\mathrm{D}}{3} \cdot\left(\mathrm{y}_{1}+4 \mathrm{y}_{2}+2 \mathrm{y}_{3}+\ldots+2 \mathrm{y}_{\mathrm{m}-1}+4 \mathrm{y}_{\mathrm{m}}+\mathrm{y}_{\mathrm{m}+1}\right)
$$

em que cada ordenada y do HU é obtida pela solução da função dentro da integral, para um valor de $\mathrm{t}-\tau$; D - passo de integração. O hidrograma de cheia foi obtido aplicando-se novamente a equação de convolução, na forma matricial, da seguinte forma:

$$
[\mathrm{Q}]=[\mathrm{h}(\mathrm{t})] \cdot[\mathrm{Pe}]
$$

em que [Q] - a matriz do hidrograma de cheia; [h(t)] matriz de ordenadas do HU e [Pe] - matriz de precipitações efetivas considerando-se a precipitação unitária igual a $1 \mathrm{~mm}$.

Para estimar os parâmetros k e n, Nash (1957) demonstrou que, para um sistema linear invariante no tempo, as seguintes relações são válidas:

$$
\begin{aligned}
& \mathrm{m} 1(\mathrm{HUI})=\mathrm{m} 1 \mathrm{~S}-\mathrm{m} 1 \mathrm{E} \\
& \mathrm{m} 2(\mathrm{HUI})=\mathrm{m} 2 \mathrm{~S}-\mathrm{m} 2 \mathrm{E}
\end{aligned}
$$

em que m1(HUI) e m2(HUI) são os primeiro e segundo momentos do HUI; m1S e m2S são os primeiro e segundo momentos do hidrograma de saída; m1E e m2E são o primeiro e o segundo momentos do hidrograma de entrada, ou seja:

$$
\begin{aligned}
\mathrm{m} 1 \mathrm{~S} & =\frac{\sum \mathrm{Q}_{\mathrm{i}} \mathrm{t}_{\mathrm{i}}}{\sum \mathrm{Q}_{\mathrm{i}}} \\
\mathrm{m} 2 \mathrm{~S} & =\frac{\sum \mathrm{Q}_{\mathrm{i}} \mathrm{t}_{\mathrm{i}}^{2}}{\sum \mathrm{Q}_{\mathrm{i}}}-\mathrm{m} 1 \mathrm{~S}^{2} \\
\mathrm{~m} 1 \mathrm{E} & =\frac{\sum \mathrm{P}_{\mathrm{i}} \mathrm{t}_{\mathrm{i}}}{\sum \mathrm{P}_{\mathrm{i}}} \\
\mathrm{m} 2 \mathrm{E} & =\frac{\sum \mathrm{P}_{\mathrm{i}} \mathrm{t}_{\mathrm{i}}^{2}}{\sum \mathrm{P}_{\mathrm{i}}}-\mathrm{m} 1 \mathrm{E}^{2}
\end{aligned}
$$

Introduzindo-se esses termos nas Eq. 11 e 12 e os resolvendo, tem-se:

$$
\begin{aligned}
& \mathrm{k}=\frac{\mathrm{m} 2 \mathrm{~S}-\mathrm{m} 2 \mathrm{E}}{\mathrm{m} 1 \mathrm{~S}-\mathrm{m} 1 \mathrm{E}} \\
& \mathrm{n}=\frac{(\mathrm{m} 1 \mathrm{~S}-\mathrm{m} 1 \mathrm{E})^{2}}{\mathrm{~m} 2 \mathrm{~S}-\mathrm{m} 2 \mathrm{E}}
\end{aligned}
$$

O modelo anteriormente descrito foi aplicado a 20 eventos de escoamento superficial observados na bacia hidrográfica estudada, transformando os valores de vazão em lâminas escoadas. Para avaliação do desempenho do Modelo Nash, usou-se o Coeficiente de Eficiência de Nash e Sutcliffe, dado pela Eq. 19, o qual, segundo Machado et al. (2003) é um dos mais importantes critérios estatísticos para avaliar a precisão de modelos hidrológicos. 


$$
\mathrm{R}^{2}=1-\frac{\sum_{\mathrm{i}=1}^{\mathrm{m}}(\text { Qobsi }- \text { Qesti })^{2}}{\sum_{\mathrm{i}=1}^{\mathrm{m}}(\text { Qesti }-\overline{\mathrm{Q}})^{2}}
$$

sendo: Qobs - vazão observada; Qest - vazão estimada pelo modelo, $\overline{\mathrm{Q}}$ - vazão média observada; $\mathrm{m}$ - número de dados no evento considerado.

$\mathrm{O}$ coeficiente $\mathrm{R}^{2}$ pode variar a partir de negativo infinito até 1 , sendo 1 um ajuste perfeito. $\mathrm{O}$ valor de $\mathrm{R}^{2}$ é fortemente influenciado por erros nas vazões máximas, razão por que, quando $\mathrm{R}^{2}$ é próximo de 1 , o modelo está obtendo bom ajuste para as cheias. O desempenho de um modelo é considerado adequado e bom se o valor de $\mathrm{R}^{2}$ supera 0,75 , e é considerado aceitável se o valor de $\mathrm{R}^{2}$ fica entre 0,36 e 0,75 (Collischonn, 2001).

Após determinados os valores de $\mathrm{k}$ e $\mathrm{n}$ pelo método dos momentos, realizou-se uma análise de sensibilidade dos dados buscando-se uma combinação melhor entre esses parâmetros, indicados pelo maior coeficiente de Nash, para cada evento analisado; para isto, um algoritmo para simulação das possíveis combinações entre $\mathrm{k}$ e $\mathrm{n}$ foi desenvolvido ajustando-se 400 situações para cada um dos eventos trabalhados. Objetivando-se encontrar uma combinação entre k e n que fosse representativa para a bacia hidrográfica, a fim de se estimar futuros hidrogramas, dividiram-se os eventos de acordo com a estação do ano determinando-se, para cada estação, a média dos parâmetros gerados pela análise de sensibilidade. Para a validação final da modelagem os parâmetros k e n médios foram aplicados a 6 eventos extraídos do verão de 2006, os quais não fizeram parte da análise anterior.

\section{RESULTADOS E DISCUSSÃO}

Apresentam-se, na Tabela 1, os valores de k e n resultantes da aplicação do método dos momentos, da análise de sensibilidade (melhor combinação de $\mathrm{k}$ e n), além da média e coeficiente de variação para os eventos, de acordo com a estação do ano. Analisando-se o coeficiente de variação pode-se dizer que ocorreu baixa variabilidade nos valores gerados para $\mathrm{k}$ e $\mathrm{n}$, haja vista a complexidade envolvida na modelagem do escoamento superficial direto demonstrando ser possível a indicação de uma combinação específica para a sub-bacia hidrográfica. Os valores de k e n médios para a bacia hidrográfica utilizados nas simulações no verão foram, respectivamente, iguais a 4,81 e 5,05, ou seja, extraídos com base nos valores obtidos pela análise de sensibilidade no ano de 2004, mais representativos que os valores de 2005, devido à maior quantidade de eventos trabalhados. Para o período outono-inverno os valores são, respectivamente, 7,58 e 4,11.

Na Figura 2 se apresentam os hidrogramas de escoamento superficial direto observados, estimados com base nos valores de $\mathrm{k}$ e n obtidos pelo método dos momentos e gerados conforme a análise de sensibilidade, ou seja, a melhor combinação dos parâmetros dentro das possíveis combinações levantadas, e hidrogramas produzidos a partir de $\mathrm{k}$ e $\mathrm{n}$ médios na respectiva estação do ano. Nesta figura, Qótimo refere-se às vazões
Tabela 1. Valores dos parâmetros k e $n$ do modelo de Nash estimados com base nos eventos de cheia na bacia hidrográfica do Ribeirão Marcela

\begin{tabular}{|c|c|c|c|c|}
\hline \multirow{2}{*}{ Estação } & \multicolumn{2}{|c|}{ Momentos } & \multicolumn{2}{|c|}{ k e n ótimos } \\
\hline & k & $n$ & k & $n$ \\
\hline \multirow{11}{*}{$\begin{array}{c}\text { Verão } \\
\text { (Ano 2004) }\end{array}$} & 2,64 & 6,95 & 3,40 & 5,85 \\
\hline & 6,23 & 5,58 & 7,44 & 4,85 \\
\hline & 5,66 & 3,88 & 7,44 & 2,97 \\
\hline & 1,64 & 9,23 & 3,4 & 5,58 \\
\hline & 3,32 & 5,79 & 2,64 & 5,58 \\
\hline & 2,72 & 8,15 & 2,64 & 8,69 \\
\hline & 6,65 & 4,42 & 7,44 & 3,78 \\
\hline & 2,65 & 8,69 & 3,4 & 7,4 \\
\hline & 3,14 & 6,39 & 5,19 & 4,43 \\
\hline & 3,4 & 3,71 & 4,23 & 2,97 \\
\hline & 4,51 & 4,33 & 5,66 & 3,45 \\
\hline \multirow{2}{*}{$\begin{array}{l}\text { média } \\
\text { CV (\%) }\end{array}$} & 3,87 & 6,10 & 4,81 & 5,05 \\
\hline & 42,7 & 32,1 & 40,2 & 36,1 \\
\hline \multirow{6}{*}{$\begin{array}{l}\text { outono/inverno } \\
\text { (Ano 2004) }\end{array}$} & 4,23 & 5,2 & 4,86 & 4,49 \\
\hline & 3,46 & 5,4 & 5,66 & 3,64 \\
\hline & 10,52 & 3,64 & 10,52 & 3,64 \\
\hline & 2,66 & 6,59 & 3,4 & 5,79 \\
\hline & 9,43 & 4,49 & 10,52 & 3,64 \\
\hline & 9,62 & 3,78 & 10,52 & 3,45 \\
\hline \multirow{2}{*}{$\begin{array}{l}\text { média } \\
\text { CV (\%) }\end{array}$} & 6,65 & 4,85 & 7,58 & 4,11 \\
\hline & 53,6 & 22,9 & 43,6 & 21,9 \\
\hline \multirow{3}{*}{$\begin{array}{c}\text { Verão } \\
\text { (Ano 2005) }\end{array}$} & 7,44 & 3,83 & 4,86 & 5,58 \\
\hline & 3,65 & 7,4 & 3,32 & 8,15 \\
\hline & 9,14 & 2,97 & 7,44 & 3,45 \\
\hline média & 6,74 & 4,73 & 5,21 & 5,73 \\
\hline CV (\%) & 41,7 & 49,6 & 40,0 & 41,1 \\
\hline
\end{tabular}

estimadas com base nos parâmetros k e n obtidos pela análise de sensibilidade, com o respectivo coeficiente de Nash - Sutcliffe; $\mathrm{Q}_{\text {médio }}$ refere-se às vazões estimadas com base nos parâmetros k e n obtidos a partir dos valores médios de k e n, com respectivo coeficiente de Nash - Sutcliffe.

Analisando-se o ajuste dos hidrogramas com base nos valores de $\mathrm{k}$ e $\mathrm{n}$ gerados pelo método dos momentos, constata-se que a maioria dos valores do coeficiente de Nash foi superior a 0,750 sendo, na média, igual a 0,853 , podendo-se considerar o modelo como de boa precisão, conforme Collischonn \& Tucci (2003). Além do coeficiente de Nash é conveniente destacar o comportamento do hidrograma estimado em relação ao observado verificando-se que, para esta situação, os hidrogramas se aproximam dos observados, mostrando boa aderência do mesmo, conforme sugerido por Mello et al. (2007).

Notou-se, de forma semelhante, nos hidrogramas gerados pela melhor combinação dos parâmetros k e n, considerável aumento de precisão das estimativas em relação aos observados, com coeficiente de Nash médio igual a 0,933; além do maior coeficiente de Nash verifica-se melhor posicionamento do deflúvio máximo dos hidrogramas simulados e do respectivo valor, características fundamentais para o modelamento de hidrogramas de cheia. Agirre et al. (2005), trabalhando com uma bacia hidrográfica do norte da Espanha e área muito semelhante à deste trabalho (470 ha), ajustaram o modelo de Nash para o HUI, verificando excelente desempenho do mesmo superando, inclusive, o modelo geomorfológico do HU, em termos do coeficiente de Nash. Fendrich (1984) trabalhou com esta 

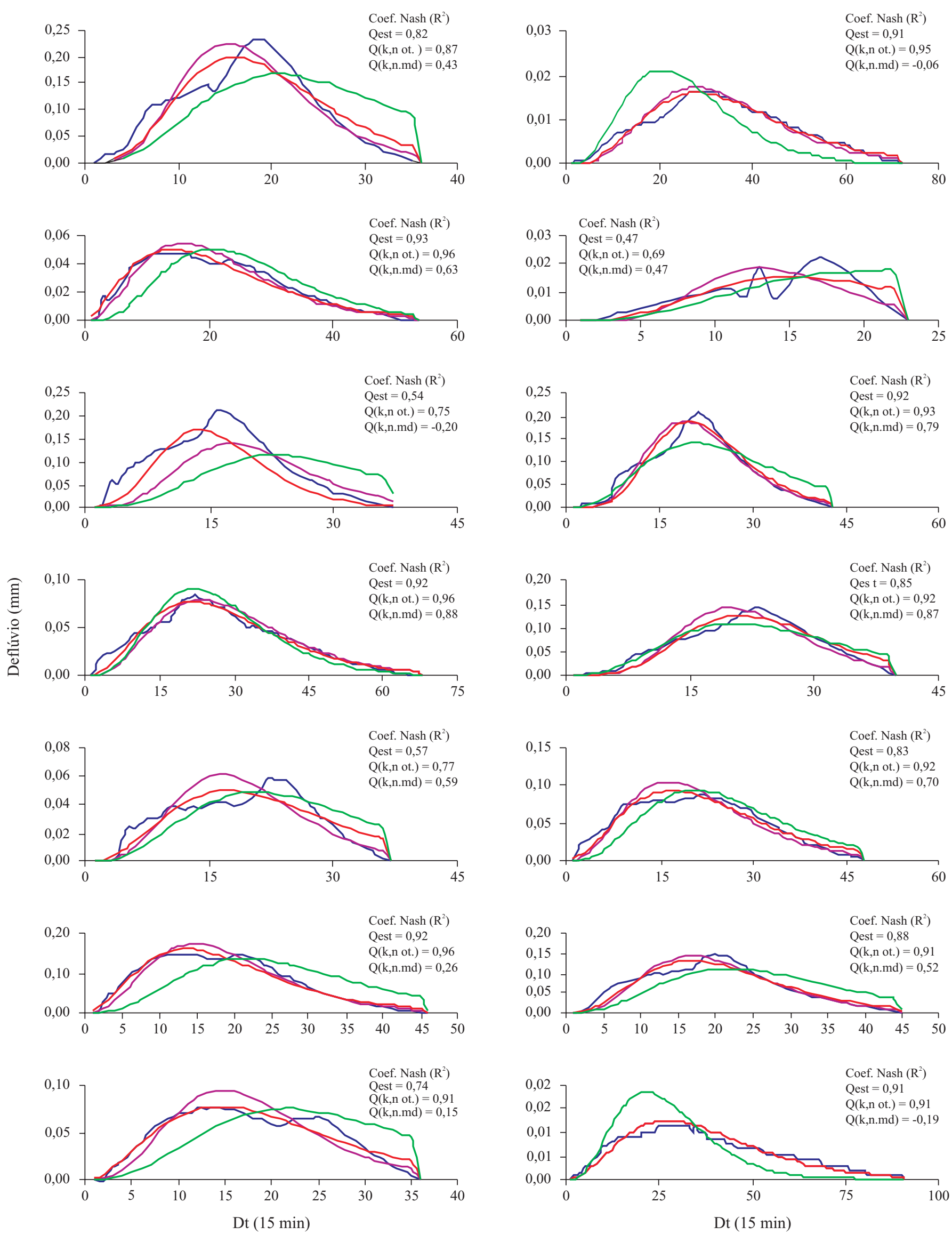

- Qobs — Qest — Q $(\mathrm{k}, \mathrm{n}$ ótimo $) \quad$ Q(k,n médio $)$ 

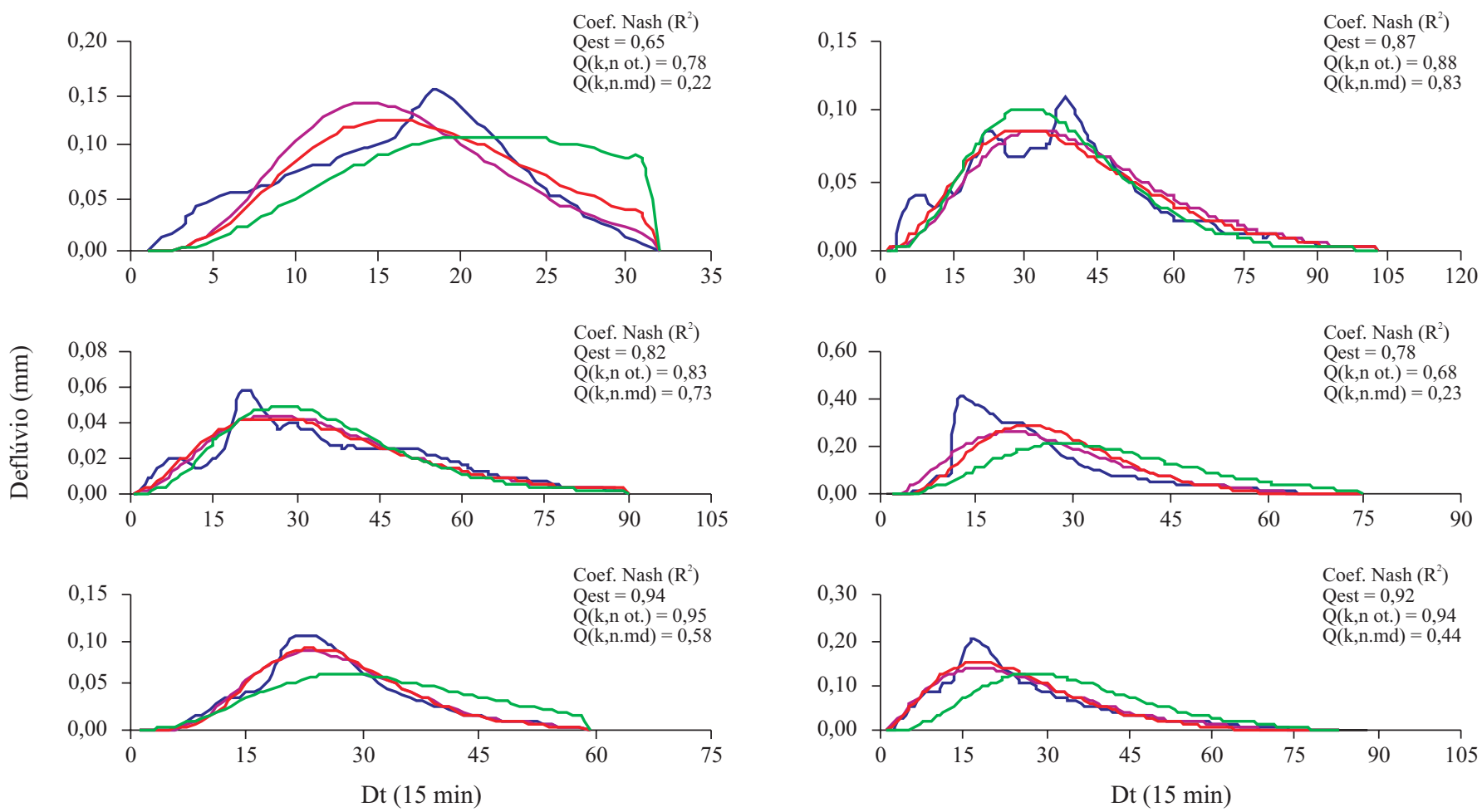

Q Qobs

Qest

Q (k,n ótimo)

Q(k,n médio)

Figura 2. Hidrogramas de cheia observados e modelados pelo modelo conceitual de Nash

modelagem para o hidrograma de cheia multimodal para uma bacia hidrográfica do estado de São Paulo com área de 610 km² e obteve resultados promissores, mesmo para uma bacia com área muito superior à do presente trabalho. Essas análises demonstram que a aplicação do método dos momentos para estimativa dos parâmetros deste modelo não produz, necessariamente, o melhor ajuste, mas gera uma estimativa inicial e aproximada do mesmo, recomendando-se uma análise de sensibilidade para pesquisar um ajuste melhor; no entanto, para cada hidrograma ocorreu uma combinação diferente dos parâmetros k e n do modelo de Nash (Tabela 1), constando-se variabilidade em torno de $40 \%$ para a série de valores estimados pelo método dos momentos. A fim de se obter uma combinação dos parâmetros k e n para a bacia hidrográfica, visando à estimativa de futuros hidrogramas de cheia, os eventos foram separados de acordo com a estação do ano e na Figura 2 os hidrogramas estão apresentados com base no valor médio de $\mathrm{k} \mathrm{e}$ n obtidos para o verão e outono/inverno, conforme a época em que ocorreram. É possível perceber redução da precisão dos hidrogramas estimados com coeficiente de Nash médio de 0,493 que, ainda assim e segundo Collischonn \& Tucci (2003), pode ser considerado como de aceitável precisão, sobretudo em se tratando de hidrogramas de cheia. Com algumas exceções, de maneira geral quando esses parâmetros são aplicados para modelagem do hidrograma, verifica-se subestimativa dos deflúvios, tanto em relação às estimativas obtidas com base nos melhores k e n (método dos momentos e análise de sensibilidade), quanto em relação aos valores observados. Em 65\% das situações analisadas constatou-se, além da redução do deflúvio máximo, superestimativa do tempo de pico do hidrograma, significando atraso na ocorrência do deflúvio máximo. Agirre et al. (2005) notaram comportamento semelhante em seus estudos e que o modelo geomorfológico se sobressaiu ligeiramente em relação ao modelo de HUI de Nash, pois o primeiro prevê a introdução de um parâmetro adicional no modelo, ligado à morfologia da bacia hidrográfica, porém a introdução de mais um parâmetro somente se justifica se houver melhoria substancial da estimativa do hidrograma dado o esforço computacional adicional e dificuldades de estimativa do mesmo, o que não ficou caracterizado pelo trabalho dos autores.

É importante analisar que alguns hidrogramas estimados, gerados pelo valor médio de $\mathrm{k}$ e $\mathrm{n}$ representativos da bacia hidrográfica, produziram coeficiente de Nash negativo (3 situações); este comportamento ocorreu porque o modelo produziu maior translação do hidrograma estimado em relação ao observado, fazendo com que houvesse um valor elevado no numerador do coeficiente (Eq. 19); entretanto, a magnitude dos deflúvios estimados não ficou comprometida com erros aceitáveis em relação aos valores observados.

Apresentam-se, na Figura 3A, gráficos que associam os deflúvios máximos observados e estimados e os tempos de pico do hidrograma observados e estimados podem ser observados na Figura 3B, ambos pelas diferentes formas de obtenção de k e n do modelo de Nash. Esses gráficos permitem visualizar a dispersão dos valores em torno da reta 1:1 e acompanhar o desempenho dos modelos no tocante a tão importantes parâmetros de caracterização do hidrograma (deflúvio máximo e tempo de ocorrência); primeiro, observa-se menor dispersão não apenas dos deflúvios máximos mas também dos tempos de pico gerados pela modelagem do hidrograma, com base nos valores de k e n produzidos pelo método dos momentos e pela análise de sensibilidade, em relação à dispersão gerada pelos 
parâmetros k e n médios do verão, demonstrando maior precisão; no entanto, verifica-se, porém, maior concentração dos pontos gerados pela média de $\mathrm{k}$ e $\mathrm{n}$ na parte inferior da reta $1: 1$, indicando que esses valores de $\mathrm{k}$ e $\mathrm{n}$ propiciam maior subestimação do deflúvio máximo; de forma inversa, nota-se maior dispersão dos valores do tempo de pico estimados pelos valores médios de $\mathrm{k}$ e $\mathrm{n}$, acima da reta 1:1, reforçando os comentários anteriores.

Para melhor caracterização da precisão da modelagem do HUI pelo modelo de Nash, os parâmetros k e n médios foram aplicados a 6 eventos extraídos do verão de 2006, os quais não fizeram parte da análise anterior e constam na Figura 4. Vê-se que em 4 situações o coeficiente de Nash obtido permite qualificar a modelagem dos hidrogramas como de aceitável a boa, sendo um dos ajustes classificados como excelente, com os coeficientes variando de 0,48 a 0,91 ; constatou-se, no entanto, em duas situações, coeficiente de Nash negativo devido ao efeito de translação.

Apresenta-se, através da Figura 5, o comportamento do coeficiente de Nash obtido pelo método dos momentos, em função
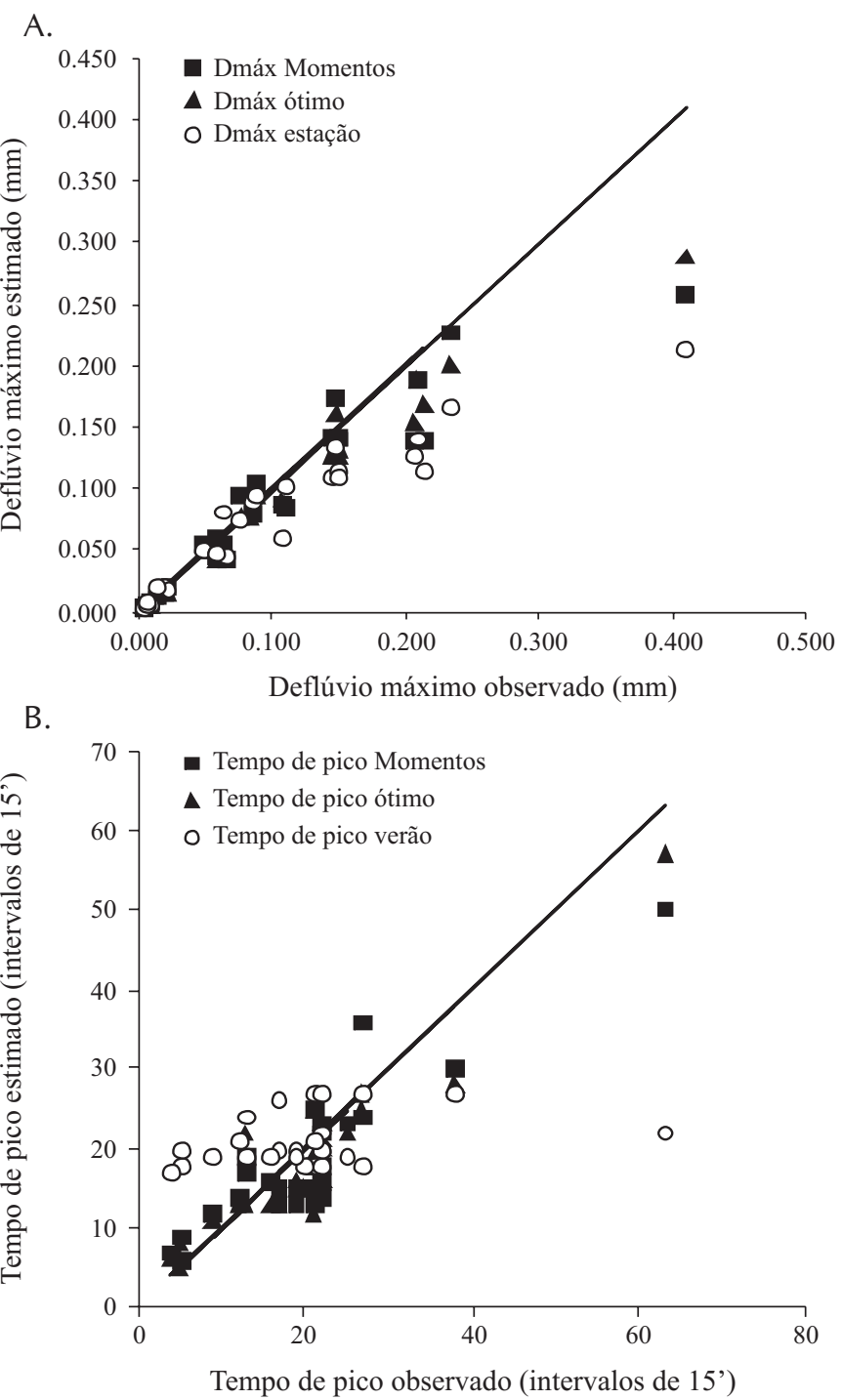

Figura 3. Dispersão dos valores de deflúvio e tempo de pico gerados com base em diferentes valores de $\mathrm{k}$ e $\mathrm{n}$
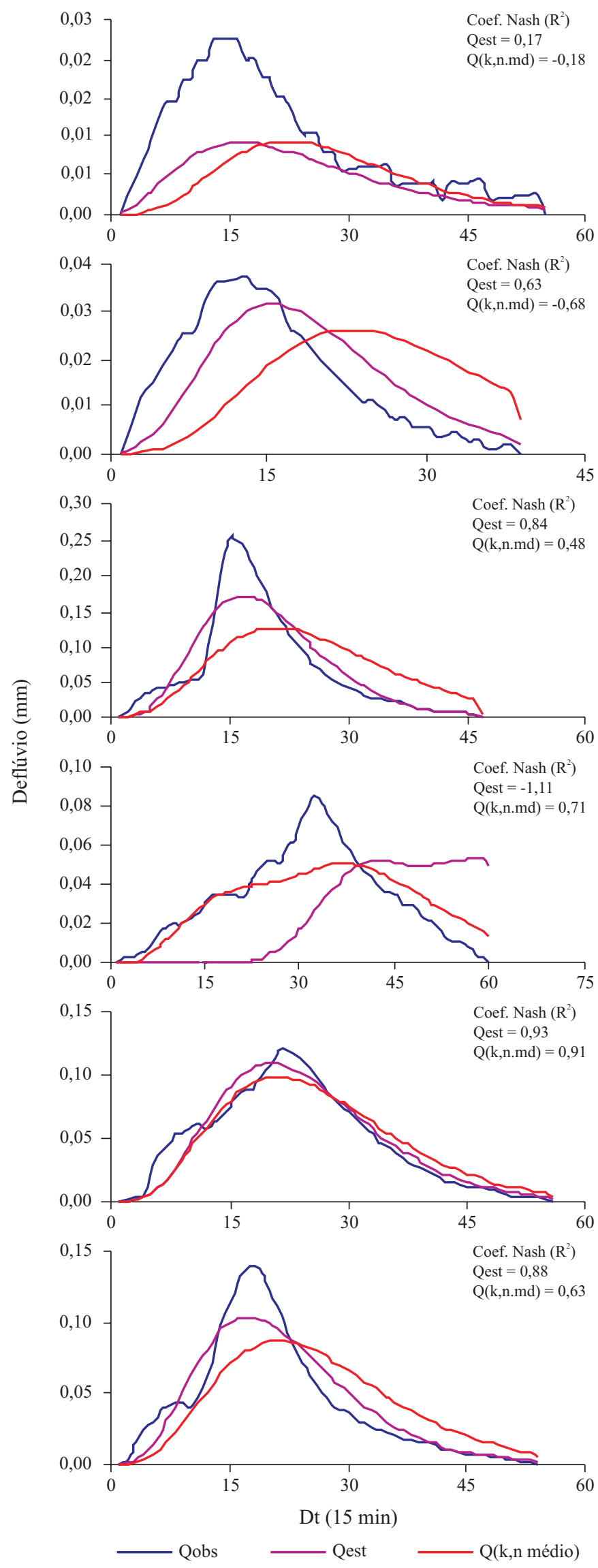

Figura 4. Hidrogramas de cheia observados e modelados com base no modelo de Nash utilizados para validação dos parâmetros propostos para a bacia hidrográfica 
do padrão dos eventos de chuva efetivos, classificados conforme Tucci (2001). Primeiro, observa-se que, quando a equação de convolução foi aplicada para 1 ou 2 eventos, a precisão dos hidrogramas estimados foi maior que para 3 eventos de precipitação efetiva, exceto para o padrão intermediário. Em termos do comportamento da precipitação, tem-se que o padrão atrasado produziu ligeiro aumento do coeficiente de Nash seguido do padrão adiantado, apesar de não se ter observado a ocorrência de 3 eventos nesta situação. Fendrich (1984) e Agirre et al. (2005), constataram que o padrão de chuva foi determinístico nas estimativas dos hidrogramas de cheia, relatando que a característica do padrão é importante devido à sua influência no comportamento da umidade do solo. Mello et al. (2007), trabalhando com modelagem da vazão de pico e deflúvio numa pequena bacia hidrográfica, demonstrou que a umidade do solo é de fundamental importância para predição do comportamento do hidrograma de cheia e sugeriu que ela seja introduzida neste processo; tais resultados, associados aos discutidos anteriormente, demonstram que, sem dúvida, o modelo de Nash produz melhores resultados em uma situação de maior umidade do solo. Wang \& Chan (1996) concluíram que a introdução de um fator associado à abstração da chuva pode proporcionar melhores resultados, sendo esta variável intimamente ligada à umidade do solo; tais observações são importantes para, em trabalhos futuros, se desenvolver e aplicar esses atributos ao processo de modelagem do hidrograma de cheia.

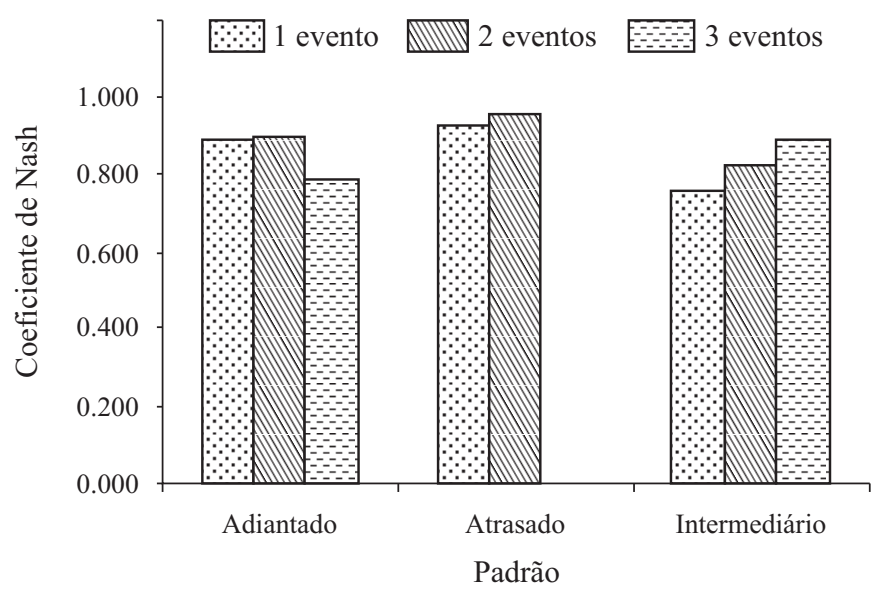

Figura 5. Distribuição do coeficiente de Nash em função do comportamento dos eventos de precipitação

De forma geral, a modelagem do hidrograma de cheia produziu bons resultados, os quais são fundamentais para estudos hidrológicos em uma bacia hidrográfica importante no contexto ambiental, em razão de ser representativa do domínio dos Latossolos, drenando diretamente para o reservatório de uma importante UHE do Sul de Minas Gerais.

\section{CONCLUSÕES}

1. O modelo de Nash mostrou-se adequado para a modelagem do escoamento de cheia na bacia hidrográfica, embora subestimando a vazão de pico.
2. A análise de sensibilidade dos parâmetros k e n propiciou melhoria na performance do modelo de Nash, mostrando que o método dos momentos sugerido originalmente pode não produzir as melhores estimativas para esses parâmetros.

3. Foi possível verificar pequena variabilidade dos parâmetros do modelo, podendo-se indicar os valores de 4,81 para k e 5,05 para n, para estimativa dos eventos de cheia durante o verão e 7,58 e 4,11 , respectivamente, para eventos do outono-inverno.

\section{LITERATURA CITADA}

Agirre, U.; Goñi, M.; López, J. J.; Gimena, F. N. Application of a unit hydrograph based on subwatershed division and comparison with Nash's instantaneous unit hydrograph. Journal of Hydrology, v.64, n.2/3, p.321-322, 2005.

Collischonn, W. Simulação hidrológica em grandes bacias. Porto Alegre: UFRGS, 2001. 194p. Tese Doutorado

Collischonn, W.; Tucci, C. E. M. Ajuste multiobjetivo dos parâmetros de um modelo hidrológico. Revista Brasileira de Recursos Hídricos, v.8, n., p.27-39, 2003.

Fendrich, R. Modelação de hidrogramas multi-modais. São Carlos: USP, 1984. 174p. Dissertação Mestrado

Machado, R. E.; Vettorazzi, C. A.; Cruciani, D. E. Simulação de escoamento em uma microbacia hidrográfica utilizando técnicas de modelagem e geoprocessamento. Revista Brasileira de Recursos Hídricos, v.8, n.1, p.147-155, 2003.

Mello, C. R; Lima, J. M.; Silva, A. M. Simulação do deflúvio e vazão de pico em microbacia hidrográfica com escoamento efêmero. Revista Brasileira de Engenharia Agrícola e Ambiental, v.11, n.4, p.410-419, 2007.

Nash, J. E. The form of instantaneous units hydrograph. In: Assemblee Generale de Toronto, 3, 1957, Toronto. Anais... Toronto: IAHS, 1957, p.114-121.

Pruski, F. F.; Brandão, V. dos S.; Silva, D. D. da. Escoamento superficial. 1.ed. Viçosa: UFV, 2003. 88p.

Quintela, A. C.; Portela, M. M. A modelação hidrológica em Portugal nos últimos 25 anos do século XX, nas perspectivas determinística, probabilística e estocástica. Revista Brasileira de Recursos Hídricos, v.7, n.4, p.51-64, 2002.

Schuler, A. E. Fluxos hidrológicos em microbacias com floresta e pastagem na Amazônia Oriental, Paragominas, Pará. Piracicaba: CENA, 2003. 120p. Tese Doutorado

Silva, A. M.; Mello, C. R. Estudos hidrológicos sobre o regime de escoamento das bacias de drenagem para o reservatório da UHE de Camargos/CEMIG. Lavras: UFLA/CEMIG, 2005. 600p. Relatório de Projeto de Pesquisa e Desenvolvimento (P\&D076).

Tucci, C. E. M. Modelos hidrológicos. Porto Alegre: UFRGS/ ABRH, 1998. 699p.

Tucci, C. E. M. Hidrologia: Ciência e aplicação. Porto Alegre: ABRH/UFRGS, 2001. 943p.

Tucci, C. E. M.; Collishonn, W. Previsão de vazão. In: Tucci, C. E. M.; Braga, B. (org.). Clima e recursos hídricos no Brasil. Porto Alegre: ABRH, 2003. p.281-348.

Wang, G.; Chen, S. A linear spatially distributed model for a surface rainfall-runoff system. Journal of Hydrology, v.185, n.1, p.183-198, 1996.

R. Bras. Eng. Agríc. Ambiental, v.12, n.3, p.258-265, 2008. 